\title{
Mechanical Losses of Polystyrenes with Different Molecular Weight Distributions
}

\author{
HILDING HögBERG, * STUART E. LOVELL, and JOHN D. FERRY
}

Department of Chemistry, University of Wisconsin, Madison, Wisconsin, U.S.A.

\begin{abstract}
Dynamic mechanical measurements have been made on two polystyrene samples with sharp molecular weight distributions and one with a broad distribution, at temperatures and frequencies corresponding to the plateau zone where the mechanical losses pass through minima. The losses are calculated in terms of the loss compliance $J^{\prime \prime}$, loss modulus $G^{\prime \prime}$, and loss tangent $\tan \delta$. For the sharp distributions, the minima in $G^{\prime \prime}$ and $\tan \delta$ increase in magnitude and shift to higher frequencies with decreasing molecular weight; the frequency dependences of all three loss functions agree approximately with the predictions of a new theory of Marvin, with the molecular weight between entanglement coupling points chosen as 24000 . The results for the broad distribution indicate that molecular weight heterogeneity increases $\tan \delta$ and $J^{\prime \prime}$ but decreases $G^{\prime \prime}$.
\end{abstract}

Tt is well known that the viscoelastic properties of polymers depend strongly Ion molecular weight distribution in the terminal zone of the time scale, where transient measurements at long times or dynamic measurements at low frequencies reflect the slowest configurational rearrangements of flexible macromolecules 1,2 . Less attention has been devoted to the effects of molecular weight and molecular weight distribution in the plateau zone, where the mechanical properties of linear polymers are dominated by entanglement coupling and a consequent transient network structure ${ }^{3-5}$.

In the plateau zone, so-called because the viscoelastic relaxation spectrum here can be approximated roughly by a plateau or box ${ }^{6,7}$ (although in many cases this is a rather crude representation), the dynamic mechanical losses pass through minima. Qualitatively these arise because at lower frequencies large losses occur from the onset of entanglement slippage and flow deformation while at high frequencies large losses occur as the transition from rubberlike to glasslike consistency is approached. At intermediate frequencies, where the entanglements do not slip but between the entanglements the chains

\footnotetext{
* Telefonaktiebolaget I. M. Ericsson, Stockholm, Sweden.
} 
Fig. 1. Mechanical losses of poly-n-octyl methacrylate in the plateau zone, plotted logarithmically against radian frequency $\omega$ at $100^{\circ} \mathrm{C}$. $G^{\prime \prime}$, loss modulus $\left(\mathrm{dyn} \theta / \mathrm{cm}^{2}\right)$; $J^{\prime \prime}$, loss compliance $\left(\mathrm{cm}^{2} /\right.$ dyne $) ; \tan \delta$, loss tangent.

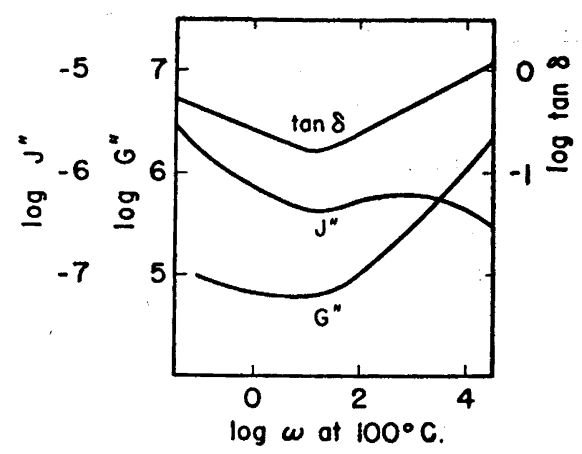

rearrange freely, the system partakes to a certain extent of the nature of an elastic network with relatively low loss.

The losses (associated with dissipation of heat in oscillating, sinusoidal mechanical deformation) can be described in various ways: ${ }^{8}$ the loss modulus $G^{\prime \prime}$ or stress $90^{\circ}$ out of phase with strain divided by strain; the loss compliance $J^{\prime \prime}$ or strain $90^{\circ}$ out of phase with stress divided by stress; or the loss tangent $\tan \delta, \delta$ being the phase angle between stress and strain. Typical examples of these functions are shown in Fig. 1 , for the plateau region of poly- $n$-octyl methacrylate. ${ }^{9}, 10$ Each shows a minimum on the frequency scale, though not at exactly the same location.

It is often supposed that mechanical properties in this region are insensitive to the molecular weight. However, the losses, especially $\tan \delta$, depend rather considerably on both molecular weight and molecular weight distribution. The present paper cites measurements on certain polystyrene samples and an interpretation in terms of a recent theory of Marvin ${ }^{11}$ to illustrate this fact.

\section{MATERIALS AND METHOD}

Two polystyrene samples (S8 and S1160) of sharp molecular weight distribution were generously furnished by Dr. H. W. McCormick of Dow Chemical Company "1. They had been prepared by anionic polymerization followed by terminating the chains with water. A third sample (19F), previously furnished also by Dow, had been prepared by conventional free radical polymerization procedures and presumably had a "most probable" distribution of molecular weights. The initial number and weight average molecular weights are given in Table 1 .

For measurements of dynamic mechanical properties at low frequencies with the torsion pendulum apparatus of Plazek, Vrancken, and Berge ${ }^{13}$, the samples were molded into discs. Those with the sharp distributions, furnished in finely divided form, were first sintered by heating in vacuo a few degrees above the softening point for several hours. Molding was performed in a press at about $100 \mathrm{lbs}$. per sq. in. at $150^{\circ} \mathrm{C}$. Measurements of characteristic frequencies and decrements of free oscillations were made as described elsewhere ${ }^{13}$ at temperatures between $125^{\circ}$ and $160^{\circ} \mathrm{C}$.

After completion of the measurements, borings were taken from the centers of the discs and their intrinsic viscosities were measured in methyl ethyl ketone. Viscosity. average molecular weights calculated therefrom ${ }^{14}$ are given in the last column of Table 1 , and indicate some but not excessive degradation during the molding and measurements. (Two other samples with sharp distributions and considerably higher molecular weights

Acta Chem. Scand. 14 (1960) No. 6 
Table 1. Initial number and weight-average molecular weights of polystyrene samples, and final viscosity-average molecular weights.

\begin{tabular}{lccc} 
& \multicolumn{2}{c}{ Initial } & Final \\
Sample & $\bar{M}_{\mathfrak{n}} \times 10^{-3}$ & $\bar{M}_{\mathbf{w}} \times 10^{-\mathbf{2}}$ & $\bar{M}_{\eta} \times 10^{-3}$ \\
S8 & 159 & 171 & 135 \\
S1160 ("60") & 279 & 286 & 240 \\
19F & 190 & 370 & 276
\end{tabular}

were also studied, but experienced more serious degradation, probably because of the longer times necessary for molding; the final $\bar{M}_{\eta}$ was only about half the initial $\bar{M}_{\mathbf{W}}$. The data from these samples are not reported.)

\section{RESULTS}

The measured characteristic frequencies and decrements were converted to the storage and loss moduli and compliances, $G^{\prime}, G^{\prime \prime}, J^{\prime}$, and $J^{\prime \prime}$, by wellknown formulas ${ }^{8,13}$. Although the range of actual frequencies was only from about 0.4 to 7 cycles/sec., the effective range was increased by reducing the measurements at all temperatures to $125^{\circ} \mathrm{C}$ with the method of reduced variables ${ }^{15}$. The shift factors $a_{\mathrm{T}}$ were calculated from the equation of Williams, Landel, and Ferry ${ }^{18}$ in the form

$$
\log a_{\mathrm{r}} \frac{9.82\left(T-T_{\mathrm{o}}\right)}{91.6+T-T_{\mathrm{o}}}
$$

with $T_{\mathrm{o}}=125.0^{\circ}$. The numerical coefficients here are derived from measurements by Grandine and Ferry ${ }^{16}$, and fit quite well also the temperature dependence of stress relaxation data of Ninomiya and Fujita ${ }^{17}$. Logarithmic plots

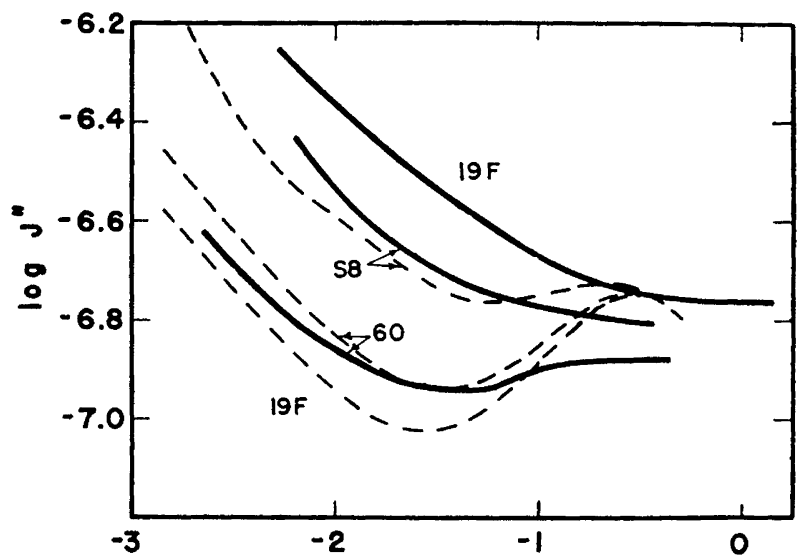

Fig. 2. Log $J^{\prime \prime}$ reduced to $125^{\circ} \mathrm{C}$., plotted against $\log \omega a_{\mathrm{r}}$ for three polystyrene samples identified by numbers; "60" is S1160. Solid curves, experimental; dashed curves, calculated from Marvin theory.

Acta Chem. Scand. 14 (1960) No. 6 


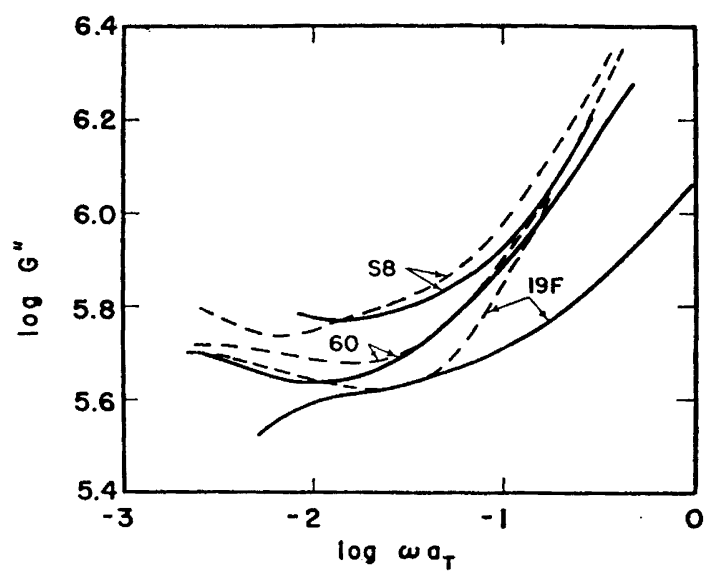

Fig. 3. Log $G^{\prime \prime}$ plotted as in Fig. 2.

of $J^{\prime}$ and $J^{\prime \prime}$ (multiplied by the usual temperature and density ratios ${ }^{15}$ ) against $\omega a_{\mathrm{r}}$ gave excellent composite curves for each sample.

The storage compliance, $J^{\prime}$, ranged from 0.25 to $1 \times 10^{-6}$, decreasing monotonically with increasing frequency. To save space, only the loss functions $J^{\prime \prime}, G^{\prime \prime}$, and $\tan \delta$ are presented here. Logarithmic plots (without points, to avoid confusion) are shown in Figs. 2, 3, and 4. Here $\omega$ is the radian frequency per second. The characteristic minima appear, and their dependence on mole-

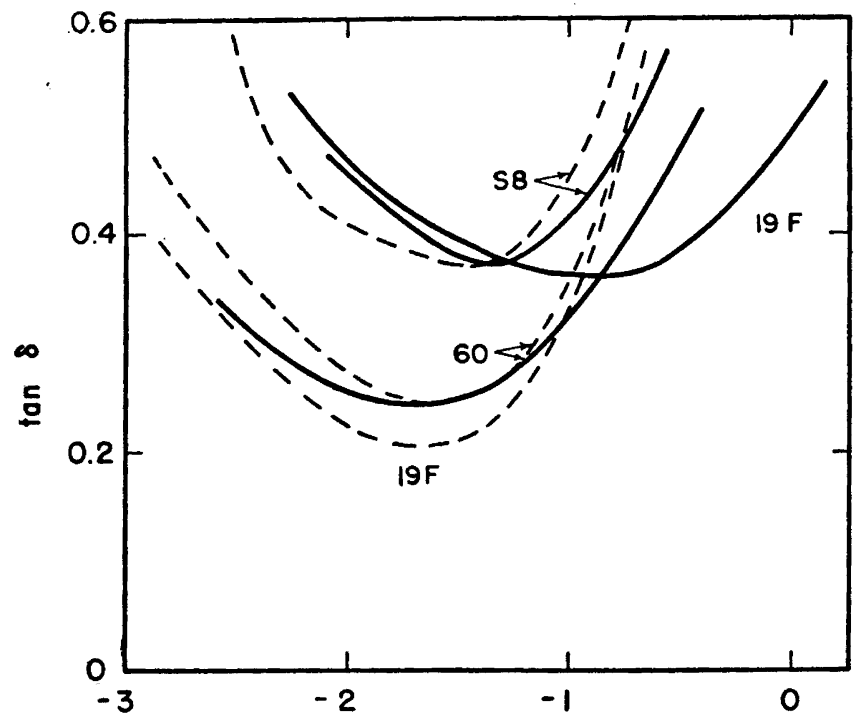

Fig. 4. Tan $\delta$ plotted as in Fig. 2.

Acta Chem. Scand. 14 (1960) No. 6 
cular weight and heterogeneity can be summarized as follows. The loss compliance increases with decreasing molecular weight and the minimum tends to shift to higher frequencies and disappear. Molecular weight heterogeneity increases the magnitude of $J^{\prime \prime}$. The loss modulus also increases with decreasing molecular weight, but the effect of heterogeneity is in the opposite direction. The loss tangent increases with decreasing molecular weight and the minimum shifts to higher frequencies. Heterogeneity also shifts the minimum to a higher magnitude and a higher frequency.

\section{DISCUSSION}

The effects illustrated here can be best interpreted with a very recent theory of Marvin ${ }^{11}$, in which viscoelastic properties in the plateau region are described by two ladder network models in series. One ladder represents a mechanical model for the Rouse-Bueche molecular theory of a cross-linked polymer, corresponding to the motions of chain segments between entanglement coupling points as reflected in measurements at high frequencies; the other represents an uncross-linked polymer with a much higher internal friction coefficient, corresponding to long-range motions which are greatly retarded by the entanglements, as reflected in measurements at very low frequencies. The Marvin theory predicts the following frequency dependence for the storage and loss moduli, expressed in slightly modified form: ${ }^{18}$

$$
G_{\mathrm{R}}^{\prime}+i G_{\mathrm{R}}^{\prime \prime}=P_{\mathrm{R}}{ }^{1 / 2} \frac{\tanh P_{\mathrm{R}}{ }^{1 / 2}+Q_{\mathrm{e}}{ }^{1 / 2} \tanh R_{\mathrm{e}} Q_{\mathrm{e}}{ }^{1 / 2} P_{\mathrm{R}}{ }^{1 / 2}}{1+Q_{\mathrm{e}}^{1 / 2} \tanh P_{\mathrm{R}}{ }^{1 / 2} \tanh R_{\mathrm{e}} Q_{\mathrm{e}}^{1 / 2} P_{\mathrm{R}}^{1 / 2}}
$$

where $G_{\mathrm{R}}^{\prime} / G^{\prime}=G_{\mathrm{R}}^{\prime \prime} / G^{\prime \prime}=1 / K_{1}$

$$
\begin{aligned}
& P_{\mathrm{R}}=i \omega_{\mathrm{R}}=i \omega K_{2} \\
& Q_{\mathrm{e}}=\left(M / 2 M_{\mathrm{e}}\right)^{2.4} \\
& R_{\mathrm{e}}=M / 2 M_{\mathrm{e}}-1 \\
& M_{\mathrm{e}}=\text { molecular weight between entanglement coupling points }
\end{aligned}
$$

The molecular interpretation of the parameters $K_{1}$ and $K_{2}$ will be mentioned later; they are both independent of $M$, but $K_{1}$ is inversely proportional to $M_{\mathrm{e}}$. Aside from this, the reduced viscoelastic properties $G_{\mathrm{R}}^{\prime}$ and $G^{\prime \prime}{ }_{\mathrm{R}}$ (also $J_{\mathrm{R}}^{\prime}$ and $J_{\mathrm{R}}^{\prime \prime}$ ) are fully determined by the ratio $M / 2 M_{\mathrm{e}}$, and $\tan \delta$ as a function of $\omega_{R}$ is determined by this ratio alone. An important equalification is that both $M$ and $M_{\mathrm{e}}$ are assumed to be devoid of heterogeneity.

To compare theory with experiment, $M_{\mathrm{e}}$ must be known. Estimates from different kinds of rheological information vary, ranging from 10000 to 30000 for polystyrene ${ }^{19}$. For the present purpose, utilization was made of the fact that the level of the minimum in $\tan \delta$, a quantity known experimentally with confidence, is a unique function of $M / 2 M_{\mathrm{e}}$. Calculations of $\tan \delta$ were made from eqn. (2) with a Bendix G-15D computer for various values of $M / 2 M_{e}$; the results near the minima are portrayed in Fig. 5. The level of the minimum 
Fig. 5.' Tan $\delta$ calculated from eqn. (2), plotted against $\log \omega_{R}$, for various values of $M / 2 M_{\mathrm{e}}$ as indicated by numbers opposite the curves.

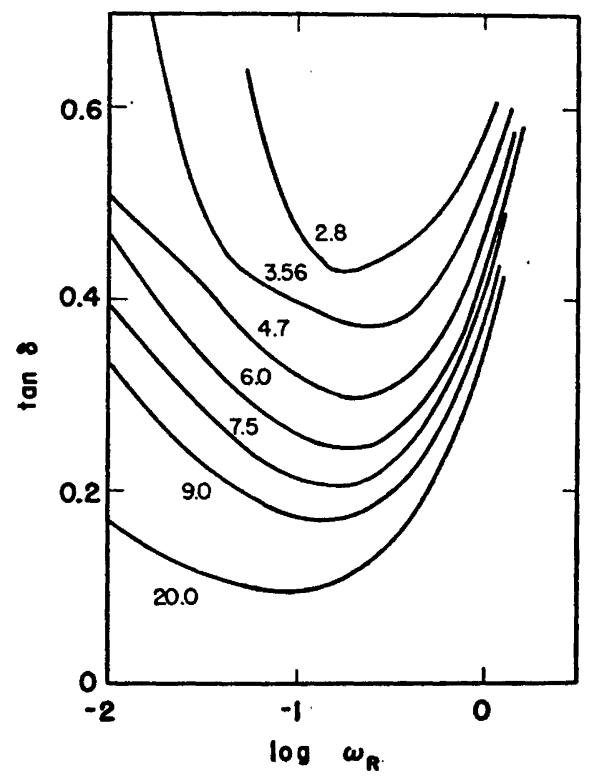

increases monotonically with decreasing $M_{\mathrm{e}}$. In fact, over the range covered it can be represented very well by the following equation:

$$
(\tan \delta)_{\min }=1.02\left(M / 2 M_{\mathrm{e}}\right)^{-0.80}
$$

Now, by substituting the experimental values for the minimum in eqn. (3), $M_{\mathrm{e}}$ can be calculated. If the weight-average values of $M$ are used, $M_{\mathrm{e}}$ is found to be 24100 and 23800 for samples S8 and S1160, respectively. Accordingly, $M_{\mathrm{e}}$ was chosen as 24000 and the functions $G^{\prime \prime}{ }_{\mathrm{R}}, J^{\prime \prime}{ }_{\mathrm{R}}$, and $\tan \delta$ were calculated from eqn. (2) for the three experimental samples.

It may be remarked that eqn. (3) provides, in principle, a very simple method for determining molecular weights of any polymer for which $M_{\mathrm{e}}$ is known. The magnitude of $\tan \delta$ can be easily measured by various experimental methods which do not require absolute calibration ${ }^{20}$. The serious limitation, of course, is the restriction to samples with sharp molecular weight distribution. Heterogeneity causes abnormally high values of $\tan \delta$ at the minimum, as seen in Fig. 4 and also in the data for the two samples which experienced degradation and consequent heterogeneity, not reported here.

The value of $K_{2}$ associated with eqn. (2) was obtained by matching the calculated and experimental minima of $\tan \delta$ on the logarithmic frequency scale, and was found to be 7.1 sec. To determine the remaining parameter $K_{1}$, logarithmic plots. of $J^{\prime \prime}{ }_{\mathrm{R}}$ and $G^{\prime \prime}{ }_{\mathrm{R}}$ were matched to the corresponding experimental plots of $J^{\prime \prime}$ and $G^{\prime \prime}$ for the sharp samples S8 and S1160; the best concurrence was obtained for $K_{1}=10^{6.38} \mathrm{~cm}^{2} /$ dyne. The theoretical predictions for $J^{\prime \prime}, G^{\prime \prime}$, and $\tan \delta$ as specified by these parameters are drawn in Figs. 2 to 4 as dashed curves.

Acta Chem. Scand. 14 (1960) No. 6 
For the two sharp samples, the respective theoretical and experimental functions agree rather well in shape, supporting the validity of the theory of Marvin as an approximate description of the viscoelastic properties in the plateau zone. For $19 \mathrm{~F}$, with a broad molecular weight distribution, the theoretical and experimental functions diverge widely. Of course, the use of $\bar{M}_{\text {}}$ for $M$ in eqn. (2) for this sample is arbitrary and in a sense meaningless. If $\bar{M}_{\mathrm{n}}$ had been used, the calculated curves would be close to those for S8. In either case, the observed loss compliance is greater than that calculated, while the observed loss modulus is smaller. It appears that viscoelastic measurements in this region could potentially yield information about molecular weight distribution; they have experimental advantages over measurements in the terminal zone, which have previously been explored for this purpose ${ }^{1,21}$, but require higher temperatures and/or longer times.

The parameter $K_{1}$ has been identified in molecular terms ${ }^{11,18}$ as a constant of the order of $\varrho R T / M_{\mathrm{e}}$, where $\varrho$ is the polymer density in $\mathrm{g} / \mathrm{cc}$. The value given above corresponds to $1.75 \varrho R T / M_{e}$, in reasonable agreement. The parameter $K_{2}$ is related to the mobility of short molecular segments. One method of formulating it in molecular terms based on the Rouse theory ${ }^{18}$ gives $K_{2}=3 a^{2} \zeta_{\mathrm{o}} M_{\mathrm{e}}^{2} / 50 M_{\mathrm{o}}^{2} k T$, where $a$ is a characteristic monomeric length, $M_{\mathrm{o}}$ is the molecular weight of a monomer unit, and $\zeta_{\mathrm{o}}$ is the translational friction coefficient per monomer unit. Substitution of the value of $K_{2}$ given above provides $\log \zeta_{0}=-1.65$ at $125^{\circ} \mathrm{C}$ (units dyne-sec/cm.) An independent calculation made from stress relaxation measurements of Fujita and Ninomiya on polystyrene ${ }^{17}$ gives $\log \zeta_{0}=-2.18$. Considering the widely different sources of information, this represents fair agreement. It may be concluded, therefore, that the Marvin theory provides a semiquantitative description not only of the relative frequency dependence of the viscoelastic losses but also of the parameters which specify their magnitudes and their positions on the frequency scale.

Relaxation $(H)$ and retardation $(L)$ spectra were also calculated from the frequency dependence of $G^{\prime \prime}$ and $J^{\prime \prime}$, and of the associated storage components $G^{\prime}$ and $J^{\prime}$, using customary approximation methods ${ }^{10}$. In each case, the agreement between calculations from the storage and loss components was very satisfactory. The spectra $H$ and $L$ show minima similar to those in $G^{\prime \prime}$ and $J^{\prime \prime}$ respectively, but shallower; over the limited range of time scale available, they do not provide enough additional information to warrant reproduction here.

Some conclusions qualitatively similar to those presented here were reported very recently by Cox, Isaksen, and Merz."

Acknowledgments. This work was supported in part by a grant from the National Science Foundation. One of us (H.H.) is pleased to acknowledge support from Statens Tekniska Forskningsråd (Swedish Technical Research Council). We are indebted to Dr. R. S. Marvin for communication of his theory before its publication, to Professor J. $O$. Hirschfelder for use of the Bendix computer, and to Joan Dell and Robert P. Cox for some technical assistance.

\section{REFERENCES}

1. Tobolsky, A. V. and Murakami, K. J. Polymer Sci. 40 (1959) 443.

2. Ferry, J. D., Williams, M. L. and Stern, D. M. J. Phys. Chem. 58 (1954) 987.

3. Mark, H. and Tobolsky, A. V. Physical Chemistry of High Polymeric Systems, Interscience Publishers, New York 1950, Chapter X and p. 344. 
4. Bueche, F. J. Chem. Phys. 20 (1952) 1979.

5. Ferry, J. D., Landel, R. F. and Williams, M. L. J. Appl. Phys. 26 (1955) 359.

6. Andrews, R. D., Hofman-Bang, N. and Tobolsky, A. V. J. Polymer Sci. 3 (1948) 669.

7. Ferry, J. D., Fitzgerald, E. R., Johnson, M. F. and Grandine, L. D., Jr. J. Appl. Phys. 22 (1951) 717.

8. Staverman, A. J. and Schwarzl, F. in Stuart, H. A. Die Physik der Hochpolymeren, Springer-Verlag, Berlin 1956, Vol. IV, Chapter I.

9. Dannhauser, W., Child, W. C., Jr. and Ferry, J. D. J. Colloid Sci. 13 (1958) 103.

10. Berge, J. W., Saunders, P. R. and Ferry, J. D. J. Colloid Sci. 14 (1959) 135.

11. Marvin, R. S., in Proc. Conf. Symp. Phenomenological Aspects of Viscoelasticity, Academic Press, New York 1960, p. 27.

12. McCormick, H. W., Brower, F. M. and Kim, L. J. Polymer Sci. 39 (1959) 87.

13. Plazek, D. J., Vrancken, M. N. and Berge, J. W. Trans. Soc. Rheology 2 (1958) 39.

14. Flory, P. J. Principles of Polymer Chemistry, Cornell University Press, Ithaca, N. Y. 1953, p. 312.

15. Ferry, J. D. and Fitzgerald, E. R. J. Colloid Sci. 8 (1953) 224.

16. Grandine, L. D., Jr. and Ferry, J. D. J. Appl. Phys. 24 (1953) 679.

17. Fujita, H. and Ninomiya, K. J. Polymer Sci. 22 (1957) 233.

18. Ferry, J. D. Viscoelastic Properties of Polymers, John Wiley and Sons, New York 1961, Chapter 10.

19. Ref. ${ }^{18}$, Chapter 13.

20. Ferry, 'J. D. in Eirich, F. R. Rheology, Academic Press, New York 1958, Vol. II; Chapter 11.

21. Watkins, J. M., Spangler, R. D. and McKannan, E. C. J. Appl. Phys. 27 (1956) 685.

22. Cox, W. P., Isaksen, R. A. and Merz, E. H., Preprints, IUPAC Symposium über Makromoleküle, Wiesbaden, 1959, Vol. 11 .

Received April 2, 1960. 